\title{
Cyclicity preserving operators on spaces of analytic functions in $\mathbb{C}^{n}$
}

\author{
Jeet Sampat
}

\begin{abstract}
For spaces of analytic functions defined on an open set in $\mathbb{C}^{n}$ that satisfy certain nice properties, we show that operators that preserve shift-cyclic functions are necessarily weighted composition operators. Examples of spaces for which this result holds true consist of the Hardy space $H^{p}\left(\mathbb{D}^{n}\right)(0<p<\infty)$, the Drury-Arveson space $\mathcal{H}_{n}^{2}$, and the Dirichlet-type space $\mathcal{D}_{\alpha}(\alpha \in \mathbb{R})$. We focus on the Hardy spaces and show that when $1 \leq p<\infty$, the converse is also true. The techniques used to prove the main result also enable us to prove a version of the Gleason-Kahane-Żelazko theorem for partially multiplicative linear functionals on spaces of analytic functions in more than one variable.
\end{abstract}

\section{Introduction}

The results presented here are motivated by a number of questions about cyclic functions in the Hardy space $H^{p}\left(\mathbb{D}^{n}\right)$. Recall that $\mathbb{D}^{n}:=\left\{z \in \mathbb{C}^{n}|| z_{i} \mid<1, \forall 1 \leq i \leq n\right\}$ is the unit polydisc in $\mathbb{C}^{n}$, and for $0<p<\infty$

$$
H^{p}\left(\mathbb{D}^{n}\right):=\left\{\left.f \in \operatorname{Hol}\left(\mathbb{D}^{n}\right)\left|\|f\|_{p}^{p}:=\sup _{0 \leq r<1} \int_{\mathbb{T}^{n}}\right| f(r w)\right|^{p} d \sigma_{n}(w)<\infty\right\}
$$

where $\sigma_{n}$ denotes the normalized Lebesgue measure on the $n$-torus $\mathbb{T}^{n}:=\left\{z \in \mathbb{C}^{n}|| z_{i} \mid=1, \forall 1 \leq i \leq n\right\}$. It is known that $H^{p}\left(\mathbb{D}^{n}\right)$ is a Banach space for all $1 \leq p<\infty$ with norm $\|\cdot\|_{p}$. A function $f \in H^{p}\left(\mathbb{D}^{n}\right)$ is said to be (shift) cyclic if

$$
\mathrm{S}[f]:=\overline{\operatorname{span}}\left\{z^{\alpha} f(z) \mid \alpha \in \mathbb{Z}^{+}(n)\right\}=\overline{\operatorname{span}}\{p f \mid p-\text { polynomial }\}=H^{p}\left(\mathbb{D}^{n}\right)
$$

where $\mathbb{Z}^{+}(n)$ is the set of all $n$-tuples $\alpha=\left(\alpha_{i}\right)_{i=1}^{n}$ of non-negative integers, and $z^{\alpha}:=z_{1}^{\alpha_{1}} z_{2}^{\alpha_{2}} \ldots z_{n}^{\alpha_{n}}$. We also have the space $H^{\infty}\left(\mathbb{D}^{n}\right)$,

$$
H^{\infty}\left(\mathbb{D}^{n}\right):=\left\{f \in \operatorname{Hol}\left(\mathbb{D}^{n}\right)\left|\|f\|_{\infty}:=\sup _{w \in \mathbb{D}^{n}}\right| f(w) \mid<\infty\right\}
$$

which is the set of all bounded analytic functions defined on $\mathbb{D}^{n}$. Just like $H^{p}\left(\mathbb{D}^{n}\right)$ for $1 \leq p<\infty, H^{\infty}\left(\mathbb{D}^{n}\right)$ is a Banach space with the supremum norm $\|\cdot\|_{\infty}$.

For $H^{p}(\mathbb{D})$ when $0<p<\infty$, cyclic functions have been characterized using Beurling's theorem and the canonical factorization theorem (see Theorem 7.4 in [3] and Theorem 4 in [4]). In the case when $n>1$, we do not have a version of Beurling's theorem nor the canonical factorization theorem (see Section 4.4 in [12] for more details). Several sufficient conditions for cyclicity in $H^{p}\left(\mathbb{D}^{n}\right)$ were provided by N. Nikolski (Theorems 3.3 and 3.4, [11]), but there are no non-trivial necessary conditions known as of now. 
One way of obtaining necessary conditions would be through operators that preserve cyclicity. That is, identify all operators $T: H^{p}\left(\mathbb{D}^{n}\right) \rightarrow H^{q}\left(\mathbb{D}^{m}\right)$ such that $T f$ is cyclic whenever $f$ is cyclic.

In the case when $p=q=2$ and $n=m=1$, a result of P. C. Gibson, M. P. Lamoureux and G. F. Margrave shows that all such operators have to be weighted composition operators and vice versa (Theorem 4, [5]). This was generalized to $H^{p}(\mathbb{D})$ for $0<p \leq \infty$ by J. Mashreghi and T. Ransford with the following theorem (Theorem 2.2, 9]).

Theorem 1.1. Let $0<p \leq \infty$ and let $T: H^{p}(\mathbb{D}) \rightarrow \operatorname{Hol}(\mathbb{D})$ be a linear map such that $T g(z) \neq 0$ for all outer functions (same as cyclic functions when $0<p<\infty) g \in H^{p}(\mathbb{D})$ and all $z \in \mathbb{D}$. Then there exist holomorphic maps $\phi: \mathbb{D} \rightarrow \mathbb{D}$ and $\psi: \mathbb{D} \rightarrow \mathbb{C} \backslash\{0\}$ such that

$$
T f=\psi \cdot(f \circ \phi)\left(\forall f \in H^{p}(\mathbb{D})\right) .
$$

It is important to note that continuity of $T$ is not assumed in the above theorem. Also, the theorem uses outer functions instead of cyclic functions but the notion of outer functions coincides with cyclic functions in the case $n=1$ and $0<p<\infty$. See Definition 4.12 for a brief discussion about outer functions. For more general spaces over $\mathbb{D}$, J. Mashreghi and T. Ransford prove the following theorem (Theorem 3.2, [9]).

Theorem 1.2. Suppose $X \subset \operatorname{Hol}(\mathbb{D})$ satisfies $(X 1)-(X 3)$ and $Y \subset X$ satisfies $(Y 1)-(Y 2)$ as defined below. Let $T: X \rightarrow \operatorname{Hol}(\mathbb{D})$ be a continuous linear map such that $T g(z) \neq 0$ for every $g \in Y$ and $z \in \mathbb{D}$. Then there exist holomorphic functions $\phi: \mathbb{D} \rightarrow \mathbb{D}$ and $\psi: \mathbb{D} \rightarrow \mathbb{C} \backslash\{0\}$ such that

$$
T f(z)=\psi(z) f(\phi(z))
$$

for each $f \in X$.

Properties $(X 1)-(X 3)$ are:

(X1) $X$ contains the set of polynomials, and they form a dense subspace of $X$.

$(X 2)$ For each $w \in \mathbb{D}$, the evaluation map $f \mapsto f(w): X \rightarrow \mathbb{C}$ is continuous.

(X3) $X$ is shift-invariant, i.e. $f \in X \Rightarrow z f \in X$.

and properties $(Y 1)-(Y 2)$ are:

$(Y 1)$ If $g \in X$ and $0<\inf _{\mathbb{D}}|g| \leq \sup _{\mathbb{D}}|g|<\infty$, then $g \in Y$.

$(Y 2)$ If $g(z)=z-\lambda$ where $\lambda \in \mathbb{T}$, then $g \in Y$.

The proof of Theorem 1.2 relies on classifying $\Lambda \in X^{*}$ such that $\Lambda(g) \neq 0, \forall g \in Y$ (Theorem 3.1, 9]). This is similar to a result known as the Gleason-Kahane-Żelazko (GKŻ) theorem (see [6] and [7]), which identifies multiplicative linear functionals in a complex unital Banach algebra through its action on invertible elements (see Theorem [5.1] in Section 5 below). In [9], it is shown that a version of the GKŻ theorem holds for modules of a complex unital Banach algebra and it can be applied to the multiplier algebra of the space $X$ satisfying properties $(X 1)-(X 3)$ above to obtain Theorem 1.2

In [8], K. Kou and J. Liu provide a similar but simpler argument for $H^{p}(\mathbb{D})$ when $1<p<\infty$, which is essentially the same as that of Theorem 1.2, but instead of the subset $Y$ they consider the set $\left\{e^{w \cdot z} \mid w \in \mathbb{C}\right\}$ 
(see Theorem 2, 8]). They also showed that the converse of Theorem [1.2 is true when $1<p<\infty$, i.e. all weighted composition operators on $H^{p}(\mathbb{D})$ for $1<p<\infty$ also preserve outer (and thus cyclic) functions.

Using techniques similar to those in [8] and [9], we can generalize Theorem 1.2 to spaces of analytic functions in more that one variable and also over arbitrary domains in a simpler way. The fundamental result presented here is the following theorem from Section 3.

Theorem 3.5. Suppose $\mathcal{X}$ satisfies $\mathbf{Q 1 - Q 3}$ over a set $\hat{D} \subset \mathbb{C}^{n}$ for some $n \in \mathbb{N}$. Let $\Lambda \in \mathcal{X}^{*}$ be such that

$$
\Lambda\left(e^{w \cdot z}\right) \neq 0 \text { for every } w \in \mathbb{C}^{n}
$$

Then, there exist $a \in \mathbb{C} \backslash\{0\}$ and $b \in \hat{D}$ such that $\Lambda(f)=a \cdot f(b)$.

Here, properties Q1-Q3 are modified versions of $(X 1)-(X 3)$ (see Section 3), so that they make sense for spaces of analytic functions in more than one variable. In Section 4, using Theorem 3.5, we will obtain the following generalization of Theorem 1.2 mentioned above.

Theorem 4.1. Suppose $\mathcal{X}$ is a space of functions on a set $\hat{D} \subset \mathbb{C}^{n}$ that are analytic in an open set $D \subset \hat{D}$, and satisfies Q1-Q3. Suppose $\mathcal{Y}$ is a topological vector space of functions on a set E such that $\Gamma_{u} g:=g(u)$, $g \in \mathcal{Y}$ defines a continuous linear functional for all $u \in E$. Let $T: \mathcal{X} \rightarrow \mathcal{Y}$ be a continuous linear operator. Then, the following are equivalent:

(1) $T\left(e^{w \cdot z}\right)$ is non-vanishing for every $w \in \mathbb{C}^{n}$.

(2) $T f(u)=a(u) f(b(u))$ for some $a \in \mathcal{Y}$ non-vanishing, and a map $b: E \rightarrow \hat{D}$.

In this case, note that $a=T(1)$ and $b=\frac{T(z)}{T(1)}$, where $T(z)$ represents the $n$-tuple of functions $\left(T\left(z_{i}\right)\right)_{i=1}^{n}$.

Using Theorem 4.1 and some other facts about Hardy spaces in several complex variables, we prove the following generalization of Theorem 1.1 above and Theorem 2 in 8 .

Theorem 4.13. (1) Fix $0<p, q<\infty$ and $m, n \in \mathbb{N}$. Let $T: H^{p}\left(\mathbb{D}^{n}\right) \rightarrow H^{q}\left(\mathbb{D}^{m}\right)$ be a bounded linear operator such that $T f$ is cyclic whenever $f$ is cyclic. Then, $T$ is necessarily a weighted composition operator, i.e. there exist analytic functions $a \in H^{q}\left(\mathbb{D}^{m}\right)$ and $b: \mathbb{D}^{m} \rightarrow \mathbb{D}^{n}$ such that $T f(z)=a(z) f(b(z))$ for every $z \in \mathbb{D}^{m}$ and $f \in H^{p}\left(\mathbb{D}^{n}\right)$.

Furthermore $a=T 1$ is cyclic and $b=\frac{T(z)}{T 1}$, where $T(z)=\left(T\left(z_{i}\right)\right)_{i=1}^{n}$.

In the case when $1 \leq q<\infty$, the converse is also true. That is, all bounded weighted composition operators from $H^{p}\left(\mathbb{D}^{n}\right)$ into $H^{q}\left(\mathbb{D}^{m}\right)$ also preserve cyclicity.

(2) Fix $0<p, q \leq \infty$ and $m, n \in \mathbb{N}$. Let $T: H^{p}\left(\mathbb{D}^{n}\right) \rightarrow H^{q}\left(\mathbb{D}^{m}\right)$ be a bounded linear operator such that Tf is outer whenever $f$ is outer. Then, $T$ is necessarily a weighted composition operator, i.e. there exist analytic functions $a \in H^{q}\left(\mathbb{D}^{m}\right)$ and $b: \mathbb{D}^{m} \rightarrow \mathbb{D}^{n}$ such that $T f(z)=a(z) f(b(z))$ for every $z \in \mathbb{D}^{m}$ and $f \in H^{p}\left(\mathbb{D}^{n}\right)$.

Furthermore, $a=T 1$ is outer and $b=\frac{T(z)}{T 1}$, where $T(z)=\left(T\left(z_{i}\right)\right)_{i=1}^{n}$.

Here, $f \in H^{p}\left(\mathbb{D}^{n}\right)$ is said to be outer if

$$
\log |f(0)|=\frac{1}{2 \pi} \int_{\mathbb{T}^{n}} \log |f| .
$$


See the discussion after Definition 4.12 below for more details on outer functions.

As mentioned before, there is a version of the GKŻ theorem for modules of complex unital Banach algebras in [9]. As an interesting byproduct of the main results presented here, we will prove the following result for Banach spaces of analytic functions, instead of modules of Banach algebras, classifying all partially multiplicative linear functionals in terms of their action on a certain set of exponentials.

Theorem 5.3. Suppose $\widehat{\mathcal{X}}$ is a space of analytic functions on a set $\widehat{D} \subset \mathbb{C}^{n}$ that satisfies Q1-Q3. Let $\Lambda \in \mathcal{X}^{*}$ such that $\Lambda(1)=1$. Then, the following are equivalent :

(i) $\Lambda\left(e^{w \cdot z}\right) \neq 0$ for every $w \in \mathbb{C}^{n}$.

(ii) $\Lambda \equiv \Lambda_{b}$ for some $b \in \widehat{D}$.

(iii) $\Lambda$ is M2.

(iv) $\Lambda$ is $M 1$.

\subsection{Acknowledgements}

This paper would not have been possible without the guidance of my $\mathrm{PhD}$ advisor, Greg Knese.

I would also like to thank John McCarthy, Brett Wick, and my colleagues Alberto Dayan and Christopher Felder for valuable discussions on topics covered in this paper and mathematics in general.

This research was partially supported by the NSF grant DMS- 1900816.

Let us start by setting up some helpful notation.

\section{Notations and preliminary results}

Fix $n \in \mathbb{N}$. For an open set $D \subset \mathbb{C}^{n}$, let $\mathcal{X} \subset \operatorname{Hol}(D)$ be a Banach space satisfying the following properties :

P1 The set of polynomials $\mathcal{P}$ is dense in $\mathcal{X}$.

P2 The point-evaluation map $\Lambda_{z}: \mathcal{X} \rightarrow \mathbb{C}$, defined as $\Lambda_{z}(f):=f(z)$ for every $f \in \mathcal{X}$, is a bounded linear functional on $\mathcal{X}$ for every $z \in D$.

P3 The $i^{t h}$-shift operator $S_{i}: \mathcal{X} \rightarrow \mathcal{X}$, defined as $S_{i} f(z):=z_{i} f(z)$ for every $\left(z_{k}\right)_{k=1}^{n}=z \in D$ and $f \in \mathcal{X}$, is a bounded linear operator for every $1 \leq i \leq n$.

Examples of spaces that satisfy P1-P3 include the Hardy space $H^{p}\left(\mathbb{D}^{n}\right)$ for $1 \leq p<\infty$, the DruryArveson space $\mathcal{H}_{n}^{2}$ on the unit ball $\mathbb{B}_{n}:=\left\{\left.z \in \mathbb{C}^{n}\left|\sum_{i=1}^{n}\right| z_{i}\right|^{2}<1\right\}$, and the Dirichlet-type spaces $\mathcal{D}_{\alpha}$ for $\alpha \in \mathbb{R}$.

$$
\begin{aligned}
\mathcal{H}_{n}^{2} & :=\left\{\left.f \in \operatorname{Hol}\left(\mathbb{B}_{n}\right)\left|\|f\|_{\mathcal{H}_{n}^{2}}^{2}:=\sum_{a \in \mathbb{Z}^{+}(n)} \frac{a_{1} ! a_{2} ! \ldots a_{n} !}{\left(a_{1}+a_{2}+\cdots+a_{n}\right) !}\right| \hat{f}(a)\right|^{2}<\infty\right\} \\
\mathcal{D}_{\alpha} & :=\left\{\left.f \in \operatorname{Hol}\left(\mathbb{D}^{n}\right)\left|\|f\|_{\mathcal{D}_{\alpha}}^{2}:=\sum_{a \in \mathbb{Z}^{+}(n)}\left(\left(a_{1}+1\right) \ldots\left(a_{n}+1\right)\right)^{\alpha}\right| \hat{f}(a)\right|^{2}<\infty\right\}
\end{aligned}
$$


The list of Dirichlet-type spaces consists of many important spaces like the usual Dirichlet space $(\alpha=1)$, the Hardy space $H^{2}\left(\mathbb{D}^{n}\right)(\alpha=0)$, and also the Bergman space $(\alpha=-1)$. For these spaces, we prove the following preliminary result.

Theorem 2.1. Suppose $\mathcal{X}$ satisfies P1-P3 over an open set $D \subset \mathbb{C}^{n}$. Let $\Lambda \in \mathcal{X}^{*}$ be such that $\Lambda\left(e^{w \cdot z}\right) \neq 0$ for every $w \in \mathbb{C}^{n}$. Then, there exist $a \in \mathbb{C} \backslash\{0\}$ and $b \in \sigma_{r}(S)$ such that $\Lambda p=a \cdot p(b)$ for every $p \in \mathcal{P}$. Here, $\sigma_{r}(S)$ is the right Harte spectrum of $S=\left(S_{i}\right)_{i=1}^{n}$.

Recall that $\sigma_{r}(S)$ is the complement in $\mathbb{C}^{n}$ of $\rho_{r}(S)$, where

$$
\rho_{r}(S):=\left\{\lambda \in \mathbb{C}^{n} \mid \exists\left\{A_{i}\right\}_{i=1}^{n} \subset \mathcal{B}(\mathcal{X}) \text { such that } \sum_{i=1}^{n}\left(S_{i}-\lambda_{i} I\right) A_{i}=I\right\} .
$$

Note that it is not immediate from P1-P3 that $e^{w \cdot z} \in \mathcal{X}$. We address this separately as a lemma before we prove Theorem 2.1.

Lemma 2.2. For each $w \in \mathbb{C}^{n}$, we have $e^{w \cdot z} \in \mathcal{X}$. In fact, $p_{k}:=\sum_{|\alpha| \leq k} \frac{w^{\alpha} \cdot z^{\alpha}}{\alpha !} \rightarrow e^{w \cdot z}$ in $\mathcal{X}$ as $k \rightarrow \infty$, where $|\alpha|:=\alpha_{1}+\cdots+\alpha_{n}$ and $\alpha !:=\alpha_{1} ! \alpha_{2} ! \ldots \alpha_{n} !$.

Proof. Fix $w \in \mathbb{C}^{n}$. We show that $\lim _{k \rightarrow \infty} p_{k}$ exists. This follows from the fact that $\mathcal{X}$ is a Banach space and

$$
\sum_{\alpha \in \mathbb{Z}^{+}(n)}\left\|\frac{w^{\alpha} \cdot z^{\alpha}}{\alpha !}\right\| \leq \sum_{\alpha \in \mathbb{Z}^{+}(n)} \frac{|w|^{\alpha}|| S||^{\alpha}|| 1||}{\alpha !}=\|1\| e^{|w| \cdot\|S\|} ;|w|=\left(\left|w_{1}\right|, \ldots,\left|w_{n}\right|\right),\|S\|=\left(\left\|S_{1}\right\|, \ldots,\left\|S_{n}\right\|\right)
$$

Let $g=\lim _{k \rightarrow \infty} p_{n}$ in $\mathcal{X}$. Note that $p_{k}$ converges to $e^{w \cdot z}$ point-wise. By P2, this implies $g(z)=e^{w \cdot z}$.

Proof of Theorem 2.1. Given that $\Lambda\left(e^{z \cdot w}\right) \neq 0$ for every $w \in \mathbb{C}^{n}$. As $\Lambda$ is continuous, we get

$$
\sum_{\alpha \in \mathbb{Z}^{+}(n)} \frac{\Lambda\left(z^{\alpha}\right) \cdot w^{\alpha}}{\alpha !} \neq 0, \forall w \in \mathbb{C}^{n}
$$

Let $\lambda_{\alpha}:=\Lambda\left(z^{\alpha}\right), \forall \alpha \in \mathbb{Z}^{+}(n)$. Now, $\left|\lambda_{\alpha}\right| \leq\left\|\Lambda\left(z^{\alpha}\right)\right\| \leq\|\Lambda\| \cdot\left\|z^{\alpha}\right\|$. Thus,

$$
\left|\lambda_{\alpha}\right| \leq\|\Lambda\| \cdot\left\|S_{1}\right\|^{\alpha_{1}} \cdot\left\|S_{2}\right\|^{\alpha_{2}} \cdots \cdot\left\|S_{n}\right\|^{\alpha_{n}} \cdot\|1\| \text { for every } \alpha \in \mathbb{Z}^{+}(n) .
$$

If we define $F(w):=\sum_{\alpha \in \mathbb{Z}^{+}(n)} \frac{\lambda_{\alpha} w^{\alpha}}{\alpha !}, \forall w \in \mathbb{C}^{n}$, then $F$ is a non-vanishing entire function such that $|F(w)| \leq\|\Lambda\| \cdot\|1\| \cdot e^{|w| \cdot|| S||}$. So, there exist $a_{0} \in \mathbb{C}$ and $b \in \mathbb{C}^{n}$ such that $F(w)=e^{a_{0}+b \cdot w}$ (see Theorem 6.1). Using the definition of $F(w)$ above and comparing power-series coefficients, we get $\lambda_{\alpha}=e^{a_{0}} b^{\alpha}, \forall \alpha \in \mathbb{Z}^{+}(n)$. Let $a:=e^{a_{0}} \in \mathbb{C} \backslash\{0\}$. This means

$$
\Lambda\left(z^{\alpha}\right)=a \cdot b^{\alpha}, \forall \alpha \in \mathbb{Z}^{+}(n) .
$$

Note that we have shown $\Lambda p=a \cdot p(b)$ for every polynomial $p$. It only remains to show that $b \in \sigma_{r}(S)$.

For the sake of contradiction, suppose $b \notin \sigma_{r}(S)$. Thus, there exists $\left\{A_{i}\right\}_{i=1}^{n} \subset \mathcal{B}(\mathcal{X})$ such that $\sum_{i=1}^{n}\left(S_{i}-\right.$ $\left.\lambda_{i} I\right) A_{i}=I$. In particular, $\sum_{i=1}^{n}\left(z_{i}-\lambda_{i}\right) A_{i} 1=1$.

Fix $\epsilon>0$ and pick $p_{i} \in \mathcal{P}$ for each $1 \leq i \leq n$ such that $\left\|A_{i} 1-p_{i}\right\|<\epsilon /\left(n \cdot\|\Lambda\| \cdot\left\|S_{i}-\lambda_{i} I\right\|\right)$. Note that,

$$
\left\|1-\sum_{i=1}^{n}\left(z_{i}-\lambda_{i}\right) p_{i}\right\|=\left\|\sum_{i=1}^{n}\left(z_{i}-\lambda_{i}\right)\left(A_{i} 1-p_{i}\right)\right\| \leq \sum_{i=1}^{n}\left\|S_{i}-\lambda_{i} I\right\| \cdot\left\|A_{i} 1-p_{i}\right\|<\sum_{i=1}^{n} \frac{\epsilon}{n \cdot\|\Lambda\|} \leq \frac{\epsilon}{\|\Lambda\|} .
$$


Based on the representation of $\Lambda$ on polynomials, we know that $\Lambda\left(\sum_{i=1}^{n}\left(z_{i}-\lambda_{i}\right) p_{i}\right)=0$. This means

$$
|a|=|\Lambda 1|=\left|\Lambda 1-\Lambda\left(\sum_{i=1}^{n}\left(z_{i}-\lambda_{i}\right) p_{i}\right)\right| \leq\|\Lambda\| \cdot \| 1-\sum_{i=1}^{n}\left(z_{i}-\lambda_{i}\right) p_{i}||<\epsilon
$$

As $\epsilon>0$ was arbitrarily chosen and $a$ is non-zero, we get a contradiction. Hence, $b \in \sigma_{r}(S)$.

Remark. It would be great if we can show that $b \in D$ but that need not be the case. It is obvious that $D \subset \sigma_{r}(S)$, but it may not be possible to extend the domain of every function in $\mathcal{X}$ to the whole of $\sigma_{r}(S)$ in order to extend the functional in the theorem to all of $\mathcal{X}$ as a point-evaluation. We shall use the notion of maximal domains to address this issue later in Section 3. For that, we mention the last part of the proof of Theorem 2.1 as a separate result.

Proposition 2.3. Suppose $\mathcal{X}$ satisfies P1-P3 over an open set $D \subset \mathbb{C}^{n}$. Let $b \in \mathbb{C}^{n}$ such that $\Lambda_{b}(p):=p(b)$ for every $p \in \mathcal{P}$ has a bounded linear extension to all of $\mathcal{X}$. Then $b$ lies in $\sigma_{r}(S)$.

Example 2.4. In the case when $\mathcal{X}=H^{p}\left(\mathbb{D}^{n}\right)$, for some $1 \leq p<\infty$, it is easy to check that $\sigma_{r}(S)=\overline{\mathbb{D}^{n}}$. So, $b$ obtained in Theorem 2.1 lies in $\overline{\mathbb{D}^{n}}$. We claim that in this case, $b$ lies in $\mathbb{D}^{n}$. For the sake of argument, assume that $b=\left(b_{i}\right)_{i=1}^{n} \in \partial \mathbb{D}^{n}$ with $b_{j} \in \mathbb{T}$ for some $1 \leq j \leq n$. Consider $q(z):=z_{j}-b_{j}$. Since $z-\beta$ is cyclic in $H^{p}(\mathbb{D})$ for all $1 \leq p<\infty$ and $\beta \notin \mathbb{D}, q$ is a cyclic polynomial in $H^{p}\left(\mathbb{D}^{n}\right)$ for all values of $p$. This means that for any given $f \in H^{p}\left(\mathbb{D}^{n}\right)$, there exist polynomials $\left\{q_{k}\right\}_{k \in \mathbb{N}}$ such that $q_{k} q \rightarrow f$. Note that

$$
\Lambda\left(q_{k} q\right)=a \cdot q_{k}(b) q(b)=0
$$

for every $k \in \mathbb{N}$ since $q(b)=0$. Thus, $\Lambda(f)=0$ which implies $\Lambda \equiv 0$, a contradiction. So, $b \in \mathbb{D}^{n}$ in this case and $\Lambda \equiv a \Lambda_{b}$.

We can make a similar argument for spaces $\mathcal{X}$ that have an envelope of cyclic polynomials over $D$. Recall that $f \in \mathcal{X}$ is cyclic if

$$
\mathrm{S}[f]:=\overline{\operatorname{span}}\left\{z^{\alpha} f(z) \mid \alpha \in \mathbb{Z}^{+}(n)\right\}=\overline{\operatorname{span}}\{p f \mid p \in \mathcal{P}\}=\mathcal{X} .
$$

Equivalently, $f \in \mathcal{X}$ is cyclic if and only if $1 \in \mathrm{S}[f]$, since polynomials form a dense subspace of $\mathcal{X}$. It is also easy to see that all cyclic functions are non-vanishing.

Definition 2.5. We say that $\mathcal{X}$ has an envelope of cyclic polynomials over $D$ if there is a family $\mathcal{F} \subset \mathcal{P}$ of cyclic polynomials such that

$$
\bigcap_{q \in \mathcal{F}}\left(\mathbb{C}^{n} \backslash \mathcal{Z}(q)\right) \subseteq D
$$

where $\mathcal{Z}(q)$ is the zero-set of $q$.

Proposition 2.6. Suppose $\mathcal{X}$ satisfies P1-P3 over an open set $D \subset \mathbb{C}^{n}$, and also has an envelope $\mathcal{F} \subset \mathcal{P}$ of cyclic polynomials. Let $\Lambda \in \mathcal{X}^{*}$ be such that $\Lambda\left(e^{w \cdot z}\right) \neq 0$ for every $w \in \mathbb{C}^{n}$. Then there exist $a \in \mathbb{C} \backslash\{0\}$ and $b \in D$ such that $\Lambda f=a \cdot f(b)$ for all $f \in \mathcal{X}$.

Proof. Getting $a \in \mathbb{C} \backslash\{0\}$ and $b \in \mathbb{C}^{n}$ such that $\Lambda(p)=a \cdot p(b)$ for every $p \in \mathcal{P}$ is the same as that in Theorem [2.1. We only need to show that $b \in D$ since in that case, $\Lambda \equiv a \Lambda_{b}$ on $\mathcal{X}$. For this, let $q \in \mathcal{F}$ 
be arbitrary and suppose $q(b)=0$. Since $q$ is cyclic, for every $f \in \mathcal{X}$ we obtain a sequence of polynomials $\left\{q_{k}\right\}_{k \in \mathbb{N}}$ such that $q_{k} q \rightarrow f$. This means that

$$
\Lambda\left(q_{k} q\right)=a \cdot q_{k}(b) q(b)=0 \rightarrow \Lambda(f)
$$

which implies $\Lambda \equiv 0$. A contradiction. Therefore $q(b) \neq 0$ for every $q \in \mathcal{F}$ and thus,

$$
b \in \bigcap_{q \in \mathcal{F}}\left(\mathbb{C}^{n} \backslash \mathcal{Z}(q)\right) \subseteq D
$$

as required.

Example 2.7. In the case of Hardy spaces $H^{p}\left(\mathbb{D}^{n}\right)$ for $1 \leq p<\infty$, we saw in Example 2.4 that the family $\mathcal{F}:=\left\{z_{i}-\beta \mid 1 \leq i \leq n\right.$ and $\left.\beta \notin \mathbb{D}\right\}$ is an envelope of cyclic polynomials over $\mathbb{D}^{n}$. The same set of polynomials also works for the Dirichlet-type spaces $\mathcal{D}_{\alpha}$ whenever $\alpha \leq 1$.

For $\alpha>1$ and $1 \leq i \leq n$, the polynomial $z_{i}-w$ with $w \in \mathbb{T}$ is not cyclic in $\mathcal{D}_{\alpha}$, and hence the same example does not work. In fact, every $f \in \mathcal{D}_{\alpha}$ is continuous up to the boundary when $\alpha>1$.

In fact $\Lambda_{b}$ is a bounded linear functional on $\mathcal{D}_{\alpha}$ even when $b \in \partial \mathbb{D}^{n}$. Thus $\mathcal{D}_{\alpha}$ cannot have an envelope of cyclic polynomials over $\mathbb{D}^{n}$. Detailed discussion on cyclicity of polynomials in the Dirichlet-type spaces can be found in [2].

Even in the case of $\mathcal{D}_{\alpha}$ when $\alpha>1$, note that the converse of Theorem 2.1 is true if we consider all $b \in \overline{\mathbb{D}^{n}}$. However, it is not obvious at all if it is the case for every space $\mathcal{X}$, since $b \in \sigma_{r}(S)$ could be arbitrary. We examine this behaviour with the help of maximal domains.

\section{Maximal domains}

Let us try to make sense of how big the domain of functions in a general space $\mathcal{X}$ that satisfies properties P1-P3 can become without losing the structure we need.

Definition 3.1. Given $\mathcal{X}$ satisfying P1-P3 over an open set $D \subset \mathbb{C}^{n}$, we define the maximal domain of functions in $\mathcal{X}$ to be the set $\hat{D}:=\left\{w \in \mathbb{C}^{n} \mid \Lambda_{w} p:=p(w), \forall p \in \mathcal{P}\right.$ has a bounded linear extension to $\left.\mathcal{X}\right\}$.

By P2, we know that $D \subset \hat{D}$. By Proposition 2.3, we know that $\hat{D} \subset \sigma_{r}(S)$. In the case of $H^{p}\left(\mathbb{D}^{n}\right)$ for $1 \leq p<\infty$ and $\mathcal{D}_{\alpha}$ for $\alpha \leq 1$, we saw in Example 2.7 that $\hat{D}=\mathbb{D}^{n}$. However for $\mathcal{D}_{\alpha}$ when $\alpha>1, \hat{D}=\overline{\mathbb{D}^{n}}$.

We shall now show that in general, $\mathcal{X}$ can be identified with a space $\hat{\mathcal{X}}$ of functions over $\hat{D}$ which also satisfies some suitable modifications of properties P1-P3. To be precise, we will show that $\mathcal{X}$ is isometrically isomorphic to a space $\hat{\mathcal{X}}$ that satisfies Q1-Q3 over $\hat{D}$ defined as follows :

Q1 The set of polynomials $\mathcal{P}$ is dense in $\hat{\mathcal{X}}$.

Q2 The point-evaluation map $\Lambda_{z}: \hat{\mathcal{X}} \rightarrow \mathbb{C}$, defined as $\Lambda_{z} f:=f(z)$ for every $f \in \hat{\mathcal{X}}$ is a bounded linear functional on $\hat{\mathcal{X}}$ for every $z \in \hat{D}$. Furthermore, if for some $z \in \mathbb{C}^{n}$ the map $\Lambda_{z} p:=p(z)$ extends to a bounded linear functional on all of $\hat{\mathcal{X}}$, then $z \in \hat{D}$.

Q3 The $i^{t h}$-shift operator $S_{i}: \hat{\mathcal{X}} \rightarrow \hat{\mathcal{X}}$, defined as $S_{i} f(z):=z_{i} f(z)$ for every $\left(z_{i}\right)_{i=1}^{n}=z \in \hat{D}$ and $f \in \hat{\mathcal{X}}$, is bounded for every $1 \leq i \leq n$. 
Q2 represents the maximality of $\hat{D}$ w.r.t bounded linear extensions of point-evaluations on polynomials.

We begin with some notation before proving the identification. For every $f \in \mathcal{X}$, define $\hat{f}(\hat{z}):=\Lambda_{\hat{z}} f$ for every $\hat{z} \in \hat{D}$ where, with the abuse of notation, we write $\Lambda_{\hat{z}} f$ to represent the extension of $\left.\Lambda_{\hat{z}}\right|_{\mathcal{P}}$ on $\mathcal{X}$ evaluated at $f$. Notice that for $z \in D, \hat{f}(z)=f(z)$ for every $f \in \mathcal{X}$. This also implies that $\left.\hat{f}\right|_{D} \in \operatorname{Hol}(D)$.

For polynomials $\hat{p} \in \hat{\mathcal{P}}$, we have that $\hat{p}(\hat{z})=p(\hat{z})$ for every $\hat{z} \in \hat{D}$. Note that $\hat{\mathcal{P}}$ is the same set as $\mathcal{P}$ but we use them differently depending on the space in which they are being considered.

Now, let $\hat{X}:=\{\hat{f}: \hat{D} \rightarrow \mathbb{C} \mid f \in \mathcal{X}\}$ and endow it with the natural vector space structure of point-wise addition and scalar multiplication. This can be done because it is obvious that $\hat{f}+\hat{g}=\widehat{f+g}$, and $\alpha \hat{f}=\widehat{\alpha f}$ for every $\alpha \in \mathbb{C}, f, g \in \mathcal{X}$.

Define the map $\iota: \mathcal{X} \rightarrow \hat{\mathcal{X}}$ as $\iota(f):=\hat{f}$ for every $f \in \mathcal{X}$. $\iota$ is clearly a vector space isomorphism, and we can define $\|\hat{f}\|_{\hat{\mathcal{X}}}:=\|f\|_{\mathcal{X}}$ for every $\hat{f} \in \hat{\mathcal{X}}$. This implies $f_{k} \rightarrow f$ in $\mathcal{X}$ if and only if $\hat{f}_{k} \rightarrow \hat{f}$ in $\hat{\mathcal{X}}$.

So, $\hat{\mathcal{X}}$ turns into a Banach space, and $\iota$ becomes an isometric isomorphism of Banach spaces. Note that since $\left.\hat{\mathcal{X}}\right|_{D}:=\left\{\left.\hat{f}\right|_{D} \mid \hat{f} \in \hat{\mathcal{X}}\right\}=\mathcal{X}$, we can say that $\hat{\mathcal{X}}$ is an extension of $\mathcal{X}$ to $\hat{D}$.

We now show that $\hat{\mathcal{X}}$ satisfies properties Q1-Q3. We start with properties Q1 and Q2.

Proposition 3.2. The space $\hat{\mathcal{X}}$ defined above satisfies $\boldsymbol{Q} \mathbf{1}$ and $\boldsymbol{Q} 2$.

Proof. In order to show Q1, first recall that $f_{k} \rightarrow f$ in $\mathcal{X}$ if and only if $\hat{f}_{k} \rightarrow \hat{f}$ in $\hat{\mathcal{X}}$. Since $\mathcal{P}$ is dense in $\mathcal{X}$ by $\mathbf{P} \mathbf{1}$, it implies easily that the set of polynomials $\hat{\mathcal{P}}$ is dense in $\hat{\mathcal{X}}$.

In order to show Q2, notice that the map $\Lambda_{\hat{z}} \hat{f}:=\hat{f}(\hat{z})$ is bounded for every $\hat{z} \in \hat{D}$ since

$$
\left|\Lambda_{\hat{z}} \hat{f}\right|=|\hat{f}(\hat{z})|=\left|\Lambda_{\hat{z}} f\right| \leq\left\|\Lambda_{\hat{z}}||_{\mathcal{X}^{*}}|| f||=\right\| \Lambda_{\hat{z}}||_{\mathcal{X}^{*}}|| \hat{f} \| .
$$

For the second part of Q2, suppose for some $\hat{z} \in \mathbb{C}^{n}, \Lambda_{\hat{z}}$ defined as above extends to all of $\hat{\mathcal{X}}$. As $\mathcal{P}$ and $\hat{\mathcal{P}}$ are identical, we can evaluate $\Lambda_{\hat{z}}$ on polynomials in $\mathcal{P}$ to get

$$
\left|\Lambda_{\hat{z}} p\right|=|p(\hat{z})|=|\hat{p}(\hat{z})| \leq\left\|\Lambda_{\hat{z}}\right\|_{\hat{\mathcal{X}}^{*}}|| \hat{p}\|\leq\| \Lambda_{\hat{z}}\left\|_{\hat{\mathcal{X}}^{*}}|| p\right\|
$$

where $\|p\|$ in the last inequality is the norm in $\mathcal{X}$. As $\mathcal{P}$ is dense in $\mathcal{X}, \Lambda_{\hat{z}}$ extends to a bounded linear functional on all of $\mathcal{X}$. By construction of $\hat{D}$, this means that $\hat{z} \in \hat{D}$.

Instead of showing that $\hat{\mathcal{X}}$ satisfies Q3 directly, we will prove a general result about multipliers which will be useful later when we consider partially multiplicative linear functionals. Recall that $\phi \in H^{\infty}(D)$ is a multiplier in $\mathcal{X}$ if $\phi f \in \mathcal{X}$ for every $f \in \mathcal{X}$. As $1 \in \mathcal{X}$, we get that $\mathcal{M}(\mathcal{X}) \subset \mathcal{X}$.

Equivalently, $\phi \in H^{\infty}(D)$ is a multiplier if multiplication by $\phi$, i.e. $M_{\phi}: \mathcal{X} \rightarrow \mathcal{X}$ defined as $M_{\phi} f=\phi f$ for every $f \in \mathcal{X}$, is a bounded linear operator on $\mathcal{X}$. The set of all multipliers in $\mathcal{X}$ forms a sub-algebra of $H^{\infty}(D)$, and is denoted by $\mathcal{M}(\mathcal{X})$. With this notation, we have the following result.

Proposition 3.3. For every $\phi \in \mathcal{M}(\mathcal{X}), \hat{\phi}$ is a multiplier in $\hat{\mathcal{X}}$. Conversely, every multiplier $\hat{\phi}$ in $\hat{\mathcal{X}}$ can be identified with some $\phi \in \mathcal{M}(\mathcal{X})$.

Proof. First, notice that for every choice of polynomials $p$ and $q$, we have that $\widehat{p} q=\hat{p} \hat{q}$. Let $f \in \mathcal{X}$ be arbitrary, and let $\left\{q_{k}\right\}_{k \in \mathbb{N}}$ be a sequence of polynomials that converges to $f$ in $\mathcal{X}$. Then for every $\hat{z} \in \hat{D}$

$$
\widehat{p f}(\hat{z})=\lim _{k \rightarrow \infty} \widehat{p q_{k}}(\hat{z})=\lim _{k \rightarrow \infty} \hat{p}(\hat{z}) \hat{q}_{k}(\hat{z})=\hat{p}(\hat{z}) \lim _{k \rightarrow \infty} \hat{q}_{k}(\hat{z})=\hat{p}(\hat{z}) \hat{f}(\hat{z}) .
$$


The first equality uses the fact that $p q_{k} \rightarrow p f \Rightarrow \widehat{p q_{k}} \rightarrow \widehat{p f}$. Thus $\widehat{p f}=\hat{p} \hat{f}$ for every $p \in \mathcal{P}, f \in \mathcal{X}$. This implies $\hat{p}$ is a multiplier in $\hat{\mathcal{X}}$ since

$$
\|\hat{p} \hat{f}\|=\|\widehat{p f}\|=\|p f\| \leq\left\|M_{p}\right\|\|f\|=\left\|M_{p}\right\|\|\hat{f}\| .
$$

Suppose now that $\phi$ is a multiplier in $\mathcal{X}$. We already know $\widehat{\phi q}=\hat{\phi} \hat{q}$ for every $q \in \mathcal{P}$. Let $f \in \mathcal{X}$ be arbitrary, and suppose again that $q_{k} \rightarrow f$ for some polynomials $q_{k}$. It is now easy to see for every $\hat{z} \in \hat{D}$,

$$
\widehat{\phi f}(\hat{z})=\lim _{k \rightarrow \infty} \widehat{\phi q_{k}}(\hat{z})=\lim _{k \rightarrow \infty} \hat{\phi}(\hat{z}) \hat{q}_{k}(\hat{z})=\hat{\phi}(\hat{z}) \lim _{k \rightarrow \infty} \hat{q}_{k}(\hat{z})=\hat{\phi}(\hat{z}) \hat{f}(\hat{z}) .
$$

Therefore $\widehat{\phi f}=\hat{\phi} \hat{f}$ for every $\phi \in \mathcal{M}(\mathcal{X}), f \in \mathcal{X}$ and so,

$$
\|\hat{\phi} \hat{f}\|=\|\widehat{\phi f}\|=\|\phi f\| \leq\left\|M_{\phi}\right\|\|f\|=\left\|M_{\phi}\right\|\|\hat{f}\| .
$$

This proves that $\hat{\phi} \in \mathcal{M}(\hat{\mathcal{X}})$ whenever $\phi \in \mathcal{M}(\mathcal{X})$.

The converse is easy since $\hat{\phi} \hat{f} \in \hat{\mathcal{X}}$ implies there exists $g \in \mathcal{X}$ such that $\hat{\phi} \hat{f}=\hat{g}$. This means $g=\left.\hat{g}\right|_{D}=\phi f$ and so, $\phi f \in \mathcal{X}$. Thus $\phi \in \mathcal{M}(\mathcal{X})$ whenever $\hat{\phi} \in \mathcal{M}(\hat{\mathcal{X}})$.

Corollary 3.3.1. The space $\hat{\mathcal{X}}$ defined above satisfies $\mathbf{Q} 3$

Proof. As the shift operators are just multiplication operators, Proposition 3.3 shows that $\hat{\mathcal{X}}$ satisfies Q3.

Now that the shift operators are bounded, we can talk about cyclic functions in $\hat{\mathcal{X}}$. However, the way we have defined the norm in $\hat{\mathcal{X}}$, it is obvious that $f \in \mathcal{X}$ is cyclic if and only if $\hat{f} \in \hat{\mathcal{X}}$ is cyclic. This and the propositions above prove the following identification theorem.

Theorem 3.4. Given a space $\mathcal{X}$ that satisfies P1-P3 over an open set $D \subset \mathbb{C}^{n}$, there exists a space $\hat{\mathcal{X}}$, consisting of functions defined over the maximal domain $\hat{D} \supseteq D$ of functions in $\mathcal{X}$, that satisfies $\mathbf{Q 1}-\mathbf{Q} 3$ and is isometrically isomorphic to $\mathcal{X}$ with the map $\iota(f):=\hat{f}$, for $f \in \mathcal{X}$.

Furthermore $\left.\hat{\mathcal{X}}\right|_{D}:=\left\{\left.\hat{f}\right|_{D} \mid \hat{f} \in \hat{\mathcal{X}}\right\}=\mathcal{X}$, and $\hat{\mathcal{X}}$ has the same set of multipliers and cyclic functions as $\mathcal{X}$. That is, $\phi \in \mathcal{M}(\mathcal{X})$ if and only if $\hat{\phi} \in \mathcal{M}(\hat{\mathcal{X}})$, and $f$ is cyclic in $\mathcal{X}$ if and only if $\hat{f}$ is cyclic in $\hat{\mathcal{X}}$.

With the help of Theorem 3.4, we can modify Theorem 2.1 as follows.

Theorem 3.5. Suppose $\mathcal{X}$ satisfies Q1-Q3 over a set $\hat{D} \subset \mathbb{C}^{n}$ for some $n \in \mathbb{N}$. Let $\Lambda \in \mathcal{X}^{*}$ be such that $\Lambda\left(e^{w \cdot z}\right) \neq 0$ for every $w \in \mathbb{C}^{n}$. Then, there exist $a \in \mathbb{C} \backslash\{0\}$ and $b \in \hat{D}$ such that $\Lambda \equiv a \Lambda_{b}$.

Proof. The proof of this theorem is the same as that of Theorem 2.1 except by property Q2, we directly obtain $b \in \hat{D}$ instead of having to show that $b \in \sigma_{r}(S)$.

It should be noted that while Theorem 3.5 is not a better result compared to Theorem [2.1, it shows that the point $b$ is not completely arbitrary; in the sense that functions in $\mathcal{X}$ are well-behaved around $b$ and that most of the structure we need can be extended to it.

In special cases like $\mathcal{D}_{\alpha}$ when $\alpha>1$, since the maximal domain is known to be different from the domain we started with, we get a better understanding of where the point $b$ lies and that it makes sense to talk about the evaluation at the point $b$ for any function in the space.

We have now finally covered all the preliminaries required to obtain cyclicity preserving operators and also prove a version of the GKŻ theorem for these spaces. 


\section{Cyclicity preserving operators}

First, we prove a generalization of Theorem 1.2 that follows easily from the previous results.

Theorem 4.1. Suppose $\mathcal{X}$ is a space of functions on a set $\hat{D} \subset \mathbb{C}^{n}$ that are analytic in an open set $D \subset \hat{D}$, and satisfies $\mathbf{Q 1 - Q 3}$. Suppose $\mathcal{Y}$ is a topological vector space of functions on a set $E$ such that $\Gamma_{u} g:=g(u)$, $g \in \mathcal{Y}$ defines a continuous linear functional for all $u \in E$. Let $T: \mathcal{X} \rightarrow \mathcal{Y}$ be a continuous linear operator. Then, the following are equivalent:

(1) $T\left(e^{w \cdot z}\right)$ is non-vanishing for every $w \in \mathbb{C}^{n}$.

(2) $T f(u)=a(u) f(b(u))$ for some $a \in \mathcal{Y}$ non-vanishing, and a map $b: E \rightarrow \hat{D}$.

In this case, note that $a=T(1)$ and $b=\frac{T(z)}{T(1)}$, where $T(z)$ represents the $n$-tuple of functions $\left(T\left(z_{i}\right)\right)_{i=1}^{n}$. Proof. $(2) \Rightarrow(1)$ is obvious.

Suppose now that (1) holds. Fix $u \in E$ and define $\Lambda:=\Gamma_{u} \circ T \in \mathcal{X}^{*}$. Note that for every $w \in \mathbb{C}^{n}$, we get

$$
\Lambda\left(e^{w \cdot z}\right)=\Gamma_{u}\left(T\left(e^{w \cdot z}\right)\right)=T\left(e^{w \cdot z}\right)(u) \neq 0
$$

since $T\left(e^{w \cdot z}\right)$ is non-vanishing by (1). By Theorem 3.5, we get that for all $f \in \mathcal{X}, \Lambda f=a(u) f(b(u))$ for some $a(u) \in \mathbb{C}$ non-zero, and $b(u) \in \hat{D}$.

Since the choice of $u \in E$ was arbitrary, we get the desired functions $a=T(1) \in \mathcal{Y}, b=\frac{T(z)}{T(1)}: E \rightarrow \hat{D}$, and that $T f(u)=a(u) f(b(u))$ for every $u \in E$.

We are now ready to identify cyclicity preserving operators. The only thing we require is the following.

Lemma 4.2. $e^{w \cdot z}$ is cyclic in $\mathcal{X}$ for every $w \in \mathbb{C}^{n}$.

Proof. Fix $w \in \mathbb{C}^{n}$. We need to find polynomials $p_{k}$ so that $\left\|p_{k} e^{w \cdot z}-1\right\| \rightarrow 0$ as $k \rightarrow \infty$. For this, let $p_{k}$ be the truncations of the power-series of $e^{-w \cdot z}$. We saw before in Lemma 2.2 that $p_{k}$ converges to $e^{-w \cdot z}$ in the norm of $\mathcal{X}$.

Next, we show that $e^{w \cdot z}$ is a multiplier. Let $q_{k}$ be the truncations of the power-series of $e^{w \cdot z}$. Given any $f \in \mathcal{X}$, we need to show $e^{w \cdot z} f$ lies in $\mathcal{X}$. Note that by the triangle inequality, we get for every $k \leq l$ that

$$
\left\|q_{l} f-q_{k} f\right\| \leq\left(\sum_{k<|\alpha| \leq l} \frac{|w|^{\alpha} \cdot\|S\|^{\alpha} \cdot\|1\|}{\alpha !}\right)\|f\| .
$$

Therefore $q_{k} f$ is a Cauchy sequence and thus, converges to some function $g \in \mathcal{X}$. As $q_{k} \rightarrow e^{w \cdot z}$ point-wise, by $\mathbf{P 2}$ we get that $q_{k} f \rightarrow e^{w \cdot z} f$ which implies $e^{w \cdot z} \in \mathcal{M}(\mathcal{X})$. This means that $M_{e^{w \cdot z}}\left(e^{-w \cdot z}\right)=1$ and thus, $M_{e^{w \cdot z}}\left(p_{k}\right) \rightarrow 1$ as $k \rightarrow \infty$. That is, $p_{k} e^{w \cdot z} \rightarrow 1$ as $k \rightarrow \infty$.

With this in mind, the following is a trivial consequence of Theorem 4.1

Theorem 4.3. Suppose $\mathcal{X}$ is a space of functions defined on a set $\hat{D} \subset \mathbb{C}^{n}$ that are analytic in an open set $D \subset \hat{D}$, and satisfies Q1-Q3. Suppose $\mathcal{Y}$ is a space of analytic functions on an open set $E \subset \mathbb{C}^{m}$ that satisfies $\boldsymbol{P} 2$ and $\boldsymbol{P} 3$. Let $T: \mathcal{X} \rightarrow \mathcal{Y}$ be such that $T f$ is cyclic whenever $f$ is cyclic. Then, there exist analytic functions $a \in \mathcal{Y}$ and $b: E \rightarrow \hat{D}$ such that $T f(u)=a(u) f(b(u))$ for every $u \in E$.

Moreover, $a=T(1)$ is cyclic and $b=\frac{T(z)}{T(1)}$ where $T(z)$ is the $n$-tuple of functions $\left(T\left(z_{i}\right)\right)_{i=1}^{n}$. 
To see how this translates to spaces $\mathcal{X}$ that satisfy properties P1-P3 instead, we get the following immediate consequence of Theorem 4.3 .

Theorem 4.4. Suppose $\mathcal{X}$ is a space of functions on an open set $D \subset \mathbb{C}^{n}$ that satisfies P1-P3. Suppose $\mathcal{Y}$ is a space of analytic functions on an open set $E \subset \mathbb{C}^{m}$ that satisfies P2 and P3. Let $T: \mathcal{X} \rightarrow \mathcal{Y}$ be such that $T f$ is cyclic whenever $f$ is cyclic. Then, there exist analytic functions $a \in \mathcal{Y}$ and $b: E \rightarrow \hat{D}$ such that $T f(u)=a(u) f(b(u))$ for every $u \in E$.

Here, $\hat{D}$ is the maximal domain for functions in $\mathcal{X}$ and with the abuse of notation, $f(b(u))=\Lambda_{b(u)} \hat{f}$. Moreover, $a=T(1)$ is cyclic and $b=\frac{T(z)}{T(1)}$ where $T(z)$ is the $n$-tuple of functions $\left(T\left(z_{i}\right)\right)_{i=1}^{n}$.

One can immediately observe in Theorems 4.1, 4.3 and 4.4 that the spaces $\mathcal{X}$ and $\mathcal{Y}$ may be defined for functions in different number of variables.

Note that for Theorems 4.3 and 4.4 we do not get a proper equivalence easily as in Theorem 4.1 since it is not at all trivial to determine when a weighted composition operator preserves cyclicity.

In the case when $\mathcal{X}=H^{p}\left(\mathbb{D}^{n}\right)$ and $\mathcal{Y}=H^{q}\left(\mathbb{D}^{m}\right)$ for some $1 \leq p<\infty$, we get that all operators that preserve cyclicity are necessarily weighted composition operators. The same is true for the Dirichlet-type spaces $\mathcal{D}_{\alpha}$ when $\alpha \leq 1$. For $\mathcal{X}=\mathcal{D}_{\alpha}$ when $\alpha>1$, we need to consider the space over its maximal domain $\hat{D}=\overline{\mathbb{D}^{n}}$. Then, all operators that preserve cyclicity will necessarily be weighted composition operators.

We now discuss the case of Hardy spaces in detail.

\subsection{Cyclicity preserving operators on Hardy spaces}

From Theorem 2 in 8 , we know that in the case of $H^{p}(\mathbb{D})$ for $1<p<\infty$, the converse of Theorem 4.3 is true. That is, all bounded weighted composition operators on $H^{p}(\mathbb{D})$ also preserve cyclicity. In the case when $n>1$, it is not obvious if all bounded weighted composition operators preserve cyclicity. We will show that the converse of Theorem 4.3 is true whenever $\mathcal{Y}=H^{q}\left(\mathbb{D}^{m}\right)$ for some $1 \leq q<\infty$. We need the following important properties of $\mathrm{S}[f]$, the shift-invariant subspace generated by a function $f \in H^{p}\left(\mathbb{D}^{n}\right)$.

Lemma 4.5. Let $f \in H^{p}\left(\mathbb{D}^{n}\right)$ for some $1 \leq p<\infty$. Then, $\phi f \in \mathrm{S}[f]$ for each $\phi \in H^{\infty}\left(\mathbb{D}^{n}\right)$.

Proof. For the sake of contradiction, let $\phi f \notin \mathrm{S}[f]$. By the Hahn-Banach theorem, there exists $\Gamma \in\left(H^{p}\left(\mathbb{D}^{n}\right)\right)^{*}$ such that $\Gamma(\phi f) \neq 0$ and $\left.\Gamma\right|_{\mathrm{S}[f]} \equiv 0$. Since $H^{p}\left(\mathbb{D}^{n}\right) \subset L^{p}\left(\mathbb{T}^{n}\right)$ is a closed subspace, by duality of $L^{p}\left(\mathbb{T}^{n}\right)$ there exists $h \in L^{p^{\prime}}\left(\mathbb{T}^{n}\right)$ such that $\Gamma(g)=\int_{\mathbb{T}^{n}} g \bar{h}$ for every $g \in H^{p}\left(\mathbb{D}^{n}\right)$, where $p^{\prime}$ is the exponent dual to $p$ (see Theorem 7.1 in $[3$, for more details).

Since $\phi$ is the weak-* limit of some sequence of analytic polynomials $p_{k}$ (take Fejér means for example) and $f \bar{h} \in L^{1}\left(\mathbb{T}^{n}\right)$ for each $f \in H^{p}\left(\mathbb{D}^{n}\right)$, we get that

$$
\Gamma(\phi f)=\int_{\mathbb{T}^{n}} \phi f \bar{h}=\lim _{k \rightarrow \infty} \int_{\mathbb{T}^{n}} p_{k} f \bar{h}=0 .
$$

The last equality follows from the fact that $p_{k} f \in \mathrm{S}[f]$ for each $k \in \mathbb{N}$, and that $\int_{\mathbb{T}^{n}} g \bar{h}=\Gamma(g)=0$ for every $g \in \mathrm{S}[f]$. Thus we reach a contradiction since $\Gamma$ was chosen so that $\Gamma(\phi f) \neq 0$.

Proposition 4.6. Let $f \in H^{p}\left(\mathbb{D}^{n}\right)$ for some $1 \leq p<\infty$. If there is a sequence $\left\{f_{k}\right\}_{k \in \mathbb{N}} \subset H^{\infty}\left(\mathbb{D}^{n}\right)$ such that $f_{k} f \rightarrow g$ as $k \rightarrow \infty$ for some $g \in \mathcal{X}$, then $g \in \mathrm{S}[f]$. In particular, if there exists a sequence $\left\{f_{k}\right\}_{k \in \mathbb{N}} \subset H^{\infty}\left(\mathbb{D}^{n}\right)$ such that $f_{k} f \rightarrow g$ for some cyclic $g \in H^{p}\left(\mathbb{D}^{n}\right)$, then $f$ is cyclic. 
Proof. The first part of the proposition follows easily from Lemma 4.5, since $f_{k} f \in \mathrm{S}[f]$ for each $k \in \mathbb{N}$, and $\mathrm{S}[f]$ is closed implies $g=\lim _{k \rightarrow \infty} f_{k} f \in \mathrm{S}[f]$.

For the second part, note that $g \in \mathrm{S}[f]$ implies $\mathrm{S}[g] \subset \mathrm{S}[f]$. Since $g$ is assumed to be cyclic, $\mathrm{S}[g]=H^{p}\left(\mathbb{D}^{n}\right)$ which means $\mathrm{S}[f]=H^{p}\left(\mathbb{D}^{n}\right)$. Therefore in this case, $f$ is also cyclic.

The following result follows easily from Theorem 4.3 and Proposition 4.6

Theorem 4.7. Suppose $\mathcal{X}$ satisfies properties Q1-Q3 over $\hat{D} \subset \mathbb{C}^{n}$. Let $T: \mathcal{X} \rightarrow H^{q}\left(\mathbb{D}^{m}\right)$ be a bounded linear map for some $1 \leq q<\infty$. Then, the following are equivalent :

(1) T preserves cyclicity, i.e. Tf is cyclic in $H^{q}\left(\mathbb{D}^{m}\right)$ whenever $f$ is cyclic in $\mathcal{X}$.

(2) There exists a cyclic function $a \in H^{q}\left(\mathbb{D}^{m}\right)$ and an analytic function $b: \mathbb{D}^{m} \rightarrow \hat{D}$ such that $T f=a \cdot(f \circ b)$ for every $f \in \mathcal{X}$.

Proof. (1) $\Rightarrow$ (2) follows from Theorem 4.3.

For the converse, let $a \in H^{q}\left(\mathbb{D}^{m}\right)$ and $b: \mathbb{D}^{m} \rightarrow \hat{D}$ be as in (2). We show that for every cyclic $f \in \mathcal{X}$, $T f=a \cdot(f \circ b)$ is cyclic in $H^{q}\left(\mathbb{D}^{m}\right)$.

Since $f$ is cyclic, there exist polynomials $p_{k}$ such that $p_{k} f \rightarrow 1$ in $\mathcal{X}$. Since $T$ is a bounded map, $T\left(p_{k} f\right) \rightarrow T(1)$ in $H^{q}\left(\mathbb{D}^{m}\right)$. Note that $T(1)=a$ is cyclic and

$$
T\left(p_{k} f\right)=a \cdot\left(p_{k} \circ b\right) \cdot(f \circ b)=\left(p_{k} \circ b\right) \cdot(a \cdot(f \circ b)) .
$$

It is easy to see $\left(p_{k} \circ b\right) \in H^{\infty}\left(\mathbb{D}^{m}\right)$ for each $n$ since the image of $b$ lies in $\hat{D} \subset \sigma_{r}(S)$. From the second part of Proposition [4.6, since $\left(p_{k} \circ b\right) \cdot(a \cdot(f \circ b)) \rightarrow a$ and $a$ is cyclic, we get that $T f=a \cdot(f \circ b)$ is cyclic in $H^{q}\left(\mathbb{D}^{m}\right)$ and thus, $(2) \Rightarrow(1)$.

Remark. (i) The proof of $(2) \Rightarrow$ (1) relies on Proposition 4.6, which further relies on the fact that the dual of $L^{p}\left(\mathbb{T}^{n}\right)$ for $1 \leq p<\infty$ is $L^{p^{\prime}}\left(\mathbb{T}^{n}\right)$ where $1 / p+1 / p^{\prime}=1$ and thus, does not translate easily to other general spaces of analytic functions.

(ii) Note that the proof of Theorem [1.1 above and Theorem 2 in [8] uses the canonical factorization theorem for Hardy spaces on the unit disc $\mathbb{D}$ (Theorem 2.8, 3 ). We do not have such a result when $n>1$ (see Section 4.2 in [12]), hence a different approach was needed.

(iii) Recall that Theorem 1.1 does not require boundedness of $T$ for the proof of (1) $\Rightarrow$ (2) to work when $\mathcal{X}=H^{p}(\mathbb{D})$. Plus, Theorem 1.1 is valid even for $0<p<1$. This is because its proof also depends on the canonical factorization theorem as mentioned above.

$(i v)$ We will see later in this section that $(1) \Rightarrow(2)$ is still valid for the case when $\mathcal{X}=H^{p}\left(\mathbb{D}^{n}\right)$ and $\mathcal{Y}=H^{q}\left(\mathbb{D}^{m}\right)$ for $0<p, q<1$ even though they are not Banach spaces. The case $p, q=\infty$ shall be treated separately as well since $H^{\infty}\left(\mathbb{D}^{n}\right)$ is not separable and hence the standard notion of cyclicity does not make any sense.

We now show that the assumption ' $T$ is a bounded operator' can be dropped in a specific case for the Hardy spaces. First, we need the following fact about boundedness of certain composition operators.

Proposition 4.8. For $1 \leq p<\infty$ and an analytic function $b: \mathbb{D}^{m} \rightarrow \mathbb{D}$, the map $T: H^{p}(\mathbb{D}) \rightarrow H^{p}\left(\mathbb{D}^{m}\right)$ defined as $T f:=f \circ b$ is a well-defined bounded linear operator. 
Proof. First, we show that $f \circ b \in H^{p}\left(\mathbb{D}^{m}\right)$ for every $f \in H^{p}(\mathbb{D})$ which shows $T$ is well-defined. The linearity of $T$ is immediate after that. We use the existence of harmonic majorants for functions in the Hardy spaces and their properties for the rest of the proof. See Section 3.2 in [12] for more details. The argument here is inspired by the one given in the corollary of Theorem 2.12 in [3] for the case $m=1$.

Let $U$ be the smallest harmonic majorant of $|f|^{p}$, i.e. the Poisson integral of $\left|f\left(e^{i \theta}\right)\right|^{p}$ given by

$$
U\left(r e^{i \theta}\right)=\frac{1}{2 \pi} \int_{0}^{2 \pi} P(r, \theta-t)\left|f\left(e^{i t}\right)\right|^{p} d t, \text { where } P(r, \theta)=\operatorname{Re}\left(\frac{1+r e^{i \theta}}{1-r e^{i \theta}}\right) .
$$

Then, $|f(u)|^{p} \leq U(u)$ for all $u \in \mathbb{D}$ which implies that $|T f(z)|^{p} \leq U(b(z))$ for every $z \in \mathbb{D}^{m}$.

Since $U$ is harmonic, $U=\operatorname{Re}(g)$ for some analytic function $g: \mathbb{D} \rightarrow \mathbb{C}$. This means that $U \circ b=\operatorname{Re}(g \circ b)$ which implies that $U \circ b$ is an $m$-harmonic function and thus, a harmonic majorant for $|T f|^{p}=|f \circ b|^{p}$. This proves that $f \circ b \in H^{p}\left(\mathbb{D}^{m}\right)$ and so, $T$ is a well-defined map. To show $T$ is bounded, observe that

$$
M_{p}(r, f \circ b)^{p} \leq U(b(0)) \leq \frac{1+|b(0)|}{1-|b(0)|} \|\left. f\right|^{p}, \text { where } M_{p}(r, f \circ b):=\left(\int_{r \mathbb{T}^{m}}|f \circ b|^{p} d \sigma_{m}\right)^{\frac{1}{p}} .
$$

The first inequality follows from the mean value property of $m$-harmonic functions, and the second inequality follows from the fact that $P(r, \theta) \leq(1+r) /(1-r)$ for all values of $r$ and $\theta$. Taking supremum over $r$ in the above inequality, we get

$$
\|f \circ b\| \leq\left(\frac{1+|b(0)|}{1-|b(0)|}\right)^{1 / p}\|f\|
$$

for every $f \in H^{p}(\mathbb{D})$ and thus, $T$ is bounded.

The following result follows easily from the previous discussion.

Proposition 4.9. Fix $1 \leq p<\infty$ and let $T: H^{p}(\mathbb{D}) \rightarrow H^{p}\left(\mathbb{D}^{m}\right)$ be a linear map such that $T 1=1$. Then, the following are equivalent:

(1) $T$ is a bounded linear map that preserves cyclicity

(2) There exists an analytic function $b: \mathbb{D}^{m} \rightarrow \mathbb{D}$ such that $T f=f \circ b$ for every $f \in H^{p}(\mathbb{D})$

Proof. As before, $(1) \Rightarrow(2)$ follows directly from Theorem 4.3.

For the converse, let $b: \mathbb{D}^{m} \rightarrow \mathbb{D}$ be an analytic function such that $T f=f \circ b$ for each $f \in H^{p}(\mathbb{D})$. By Proposition 4.8. $T$ is a bounded linear operator. By $(2) \Rightarrow(1)$ in Theorem $4.7 . T$ preserves cyclicity.

Remark. Note that the only place we use that the domain of $H^{p}(\mathbb{D})$ is in one variable, is to show boundedness of $f \mapsto f \circ b$ for every $b: \mathbb{D}^{m} \rightarrow \mathbb{D}$. More precisely, we use the fact that any harmonic function $U$ in one variable is the real part of some holomorphic function. This is not true for $n>1$ (see Section 2.4 in [12]).

As mentioned in Remark (iv) under Theorem [4.7, we now consider the cases $0<p<1$ and $p=\infty$.

Example 4.10. First, we address the case $\mathcal{X}=H^{p}\left(\mathbb{D}^{n}\right)$ for $0<p<1$. Fix $0<p<1$ and note that $H^{p}\left(\mathbb{D}^{n}\right)$ satisfies P1-P3 if we replace boundedness with continuity. The issue is that $H^{p}\left(\mathbb{D}^{n}\right)$ is not a Banach space. Even though $H^{p}\left(\mathbb{D}^{n}\right)$ is not normable, it is still a complete metric space under $d_{p}(f, g):=\|f-g\|_{p}^{p}$ where $\|\cdot\|_{p}$ is as defined in Section 1. 
Using this, its bounded linear functionals can be defined in the usual manner. That is, we say that $\Lambda: H^{p}\left(\mathbb{D}^{n}\right) \rightarrow \mathbb{C}$ is bounded if

$$
\|\Lambda\|:=\sup _{\|f\|_{p}=1}|\Lambda(f)|<\infty
$$

This means that $|\Lambda(f)| \leq\|\Lambda\| \cdot\|f\|$ for all bounded $\Lambda$, and $f \in H^{p}\left(\mathbb{D}^{n}\right)$. It is easy to verify that this notion of boundedness is equivalent to the continuity of $\Lambda$. Similarly, we say an operator $T: H^{p}\left(\mathbb{D}^{n}\right) \rightarrow H^{q}\left(\mathbb{D}^{m}\right)$ for some $0<q \leq \infty$ is bounded if

$$
\|T\|:=\sup _{\|f\|_{p}=1}\|T f\|_{q}<\infty .
$$

As was the case with linear functionals, it is easy to verify that this notion of boundedness is equivalent to the continuity of $T$. This implies that for every bounded linear operator $T$ on $H^{p}\left(\mathbb{D}^{n}\right)$ and $f \in H^{p}\left(\mathbb{D}^{n}\right)$, $\|T f\| \leq\|T\| \cdot\|f\|$. This means Lemmas 2.2 and 4.2 hold for $\mathcal{X}=H^{p}\left(\mathbb{D}^{n}\right)$ even when $0<p<1$.

In order to show that Theorem 4.3 holds for $\mathcal{X}=H^{p}\left(\mathbb{D}^{n}\right)$, we only need to show that Theorem 3.5 holds since the arguments in the proof of Theorem 4.3 do not rely on the Banach space structure of $\mathcal{X}$ except when Theorem 3.5 is applied. First, we show that the maximal domain for functions in $H^{p}\left(\mathbb{D}^{n}\right)$ when $0<p<1$ is also $\mathbb{D}^{n}$.

Fix $0<p<1$. We will show as in Example 2.7 that the family $\mathcal{F}:=\left\{z_{i}-\beta \mid 1 \leq i \leq n\right.$ and $\left.\beta \notin \mathbb{D}\right\}$ is an envelope of cyclic polynomials in $H^{p}\left(\mathbb{D}^{n}\right)$. Let $b \in \overline{\mathbb{D}^{n}}$ be such that $\left.\Lambda_{b}\right|_{\mathcal{P}}$ extends to a bounded linear functional $\Lambda \in H^{p}\left(\mathbb{D}^{n}\right)$. Thus $b_{j} \in \mathbb{T}$ for some $1 \leq j \leq n$.

Using subordination and an argument similar to Theorem 3.2 in $\left[3\right.$, we can show that $1 /\left(z-b_{j}\right) \in H^{p}(\mathbb{D})$. Consider a sequence of polynomials $\left\{q_{k}\right\}_{k \in \mathbb{N}}$ such that $q_{k} \rightarrow 1 /\left(z-b_{j}\right)$ in $H^{p}(\mathbb{D})$ and note that $q_{k}\left(z-b_{j}\right) \rightarrow 1$ since multiplication by $z-b_{j}$ is a bounded linear operator on $H^{p}(\mathbb{D})$. This implies $z-b_{j}$ is cyclic in $H^{p}(\mathbb{D})$ and thus, $q(z):=z_{j}-b_{j}$ is cyclic in $H^{p}\left(\mathbb{D}^{n}\right)$. Clearly $\mathcal{F}$ defined above is then an envelope of cyclic polynomials. This means that for any given $f \in H^{p}\left(\mathbb{D}^{n}\right)$, there exists a sequence of polynomials $\left\{p_{k}\right\}_{k \in \mathbb{N}}$ such that $p_{k} q \rightarrow f$. Since $q(b)=0$, we get

$$
\Lambda(f)=\lim _{k \rightarrow \infty} \Lambda\left(p_{k} q\right)=\lim _{k \rightarrow \infty} p_{k}(b) q(b)=0 .
$$

This means $\Lambda \equiv 0$, a contradiction. So, $b \in \mathbb{D}^{n}$ and we get $\hat{D}=\mathbb{D}^{n}$ for $H^{p}\left(\mathbb{D}^{n}\right)$ when $0<p<1$.

It is known that all outer functions in $H^{p}(\mathbb{D})$ are also cyclic for $0<p<1$ (Theorem 4, [4) but we prove $z-b_{j}$ is cyclic differently to show one way of obtaining an envelope of cyclic polynomials in the case when $\mathcal{X}$ is not a Banach space. Notice that the only other place we use the norm in the proof of Theorem 2.1 (and hence Theorem [3.5) is to obtain the non-vanishing entire function $F(w):=\sum_{\alpha \in \mathbb{Z}^{+}(n)} \frac{\Lambda\left(z^{\alpha}\right) \cdot w^{\alpha}}{\alpha !}$ using

$$
\left|\Lambda\left(z^{\alpha}\right)\right| \leq\|\Lambda\| \cdot\left\|z^{\alpha}\right\| \leq\|\Lambda\| \cdot\left\|S_{1}\right\|^{\alpha_{1}} \ldots\left\|S_{n}\right\|^{\alpha_{n}} \cdot\|1\| \text {, for every } \alpha \in \mathbb{Z}^{+}(n) .
$$

As we saw above, this should not be an issue for $H^{p}\left(\mathbb{D}^{n}\right)$ since $\|\Lambda\|$ makes just as much sense and $\left\|z^{\alpha}\right\|=1$ for all $\alpha \in \mathbb{Z}^{+}(n)$. This gives us $\left|\Lambda\left(z^{\alpha}\right)\right| \leq\|\Lambda\|$, which is good enough for the rest of the proof to work. Therefore Theorem 3.5 holds for $\mathcal{X}=H^{p}\left(\mathbb{D}^{n}\right)$ and $\mathcal{Y}=H^{q}\left(\mathbb{D}^{m}\right)$, and so does Theorem 4.3 even when $0<p, q<1$.

Example 4.11. The case $p=\infty$ is a little different since the problem here is that the set of polynomials is not dense in $H^{\infty}\left(\mathbb{D}^{n}\right)$. In fact $H^{\infty}\left(\mathbb{D}^{n}\right)$ is not separable so cyclicity of functions does not make sense. However in the case when $n=1$, we know that all cyclic functions in $H^{p}(\mathbb{D})$ for $0<p<\infty$ are outer 
functions and vice versa (Theorem 7.4, [3], Theorem 4, 4]). Since outer functions do make sense for $n \geq 1$ and $p=\infty$, we can talk about outer functions instead of cyclic functions in this case.

Definition 4.12. For $0<p \leq \infty$, a function $f \in H^{p}\left(\mathbb{D}^{n}\right)$ is said to be outer in $H^{p}\left(\mathbb{D}^{n}\right)$ if $\log |f(0)|=$ $\int_{\mathbb{T}^{n}} \log |f|$.

It is known that the class of outer functions is different from cyclic functions when $n>1$. In fact, all cyclic functions are outer, but there are outer functions that are not cyclic. The proof of this fact, and more details on outer functions can be found in Section 4.4 of [12. The argument uses different composition operators between $H^{2}\left(\mathbb{D}^{2}\right)$ and a few different Hardy spaces on the unit disc to explicitly find such a function. This was a motivating result for studying operators that preserve outer/cyclic functions with the hope of getting a better understanding of the difference between outer and cyclic functions. We discuss this a little bit in Theorem 4.14 below.

Note that in the case $p=\infty$, the hypothesis of Theorem 3.5 does not make sense. In fact, we will completely avoid using maximal domains for $H^{\infty}\left(\mathbb{D}^{n}\right)$ since without cyclicity, we cannot even determine if $\hat{D} \subset \sigma_{r}(S)$. Instead, consider $\Lambda \in\left(H^{\infty}\left(\mathbb{D}^{n}\right)\right)^{*}$ such that $\Lambda(f) \neq 0$ for all outer functions $f \in H^{\infty}\left(\mathbb{D}^{n}\right)$. Since $e^{w \cdot z}$ is clearly an outer function for all $w \in \mathbb{C}^{n}$, we proceed as in the proof of Theorem 2.1 to obtain $\left.\left.\Lambda\right|_{\mathcal{P}} \equiv a \Lambda_{b}\right|_{\mathcal{P}}$ for some $a \in \mathbb{C} \backslash\{0\}$ and $b \in \mathbb{C}^{n}$.

Now, proceed as in Example 2.7 and instead of having an envelope of cyclic polynomials, we now have an envelope of outer polynomials which is the same set of polynomials $\mathcal{F}=\left\{z_{i}-\beta \mid 1 \leq i \leq n, \beta \notin \mathbb{D}\right\}$. Since $\Lambda(f) \neq 0$ for all outer functions $f$, we get $b_{i}-\beta \neq 0$ for all $1 \leq i \leq n$ and $\beta \notin \mathbb{D}$ which implies $b_{i} \in \mathbb{D}$ for every $1 \leq i \leq n$. Therefore, $b \in \mathbb{D}^{n}$ and $\Lambda f=a \cdot f(b)$ for all $f \in H^{\infty}\left(\mathbb{D}^{n}\right)$.

Thus, the conclusion of Theorem 2.1 is valid for $H^{\infty}\left(\mathbb{D}^{n}\right)$ if we consider $\Lambda \in\left(H^{\infty}\left(\mathbb{D}^{n}\right)\right)^{*}$ that acts on outer functions as above and so, Theorem 4.4 is valid for $\mathcal{X}=H^{\infty}\left(\mathbb{D}^{n}\right)$ if we replace cyclic functions with outer functions and $\hat{D}$ with $\mathbb{D}^{n}$. A similar logic can be applied to operators that preserve outer functions in $H^{p}\left(\mathbb{D}^{n}\right)$ for $0<p<\infty$.

This discussion about Hardy spaces above yields the following generalization of Theorem 1.1 for bounded operators from $H^{p}\left(\mathbb{D}^{n}\right)$ into $H^{q}\left(\mathbb{D}^{m}\right)$ for all $0<p, q \leq \infty$ and $m, n \in \mathbb{N}$ that preserve cyclic/outer functions.

Theorem 4.13. (1) Fix $0<p, q<\infty$ and $m, n \in \mathbb{N}$. Let $T: H^{p}\left(\mathbb{D}^{n}\right) \rightarrow H^{q}\left(\mathbb{D}^{m}\right)$ be a bounded linear operator such that $T f$ is cyclic whenever $f$ is cyclic. Then, $T$ is necessarily a weighted composition operator, i.e. there exist analytic functions $a \in H^{q}\left(\mathbb{D}^{m}\right)$ and $b: \mathbb{D}^{m} \rightarrow \mathbb{D}^{n}$ such that $T f(z)=a(z) f(b(z))$ for every $z \in \mathbb{D}^{m}$ and $f \in H^{p}\left(\mathbb{D}^{n}\right)$.

Furthermore $a=T 1$ is cyclic and $b=\frac{T(z)}{T 1}$, where $T(z)=\left(T\left(z_{i}\right)\right)_{i=1}^{n}$.

In the case when $1 \leq q<\infty$, the converse is also true. That is, all bounded weighted composition operators from $H^{p}\left(\mathbb{D}^{n}\right)$ into $H^{q}\left(\mathbb{D}^{m}\right)$ also preserve cyclicity.

(2) Fix $0<p, q \leq \infty$ and $m, n \in \mathbb{N}$. Let $T: H^{p}\left(\mathbb{D}^{n}\right) \rightarrow H^{q}\left(\mathbb{D}^{m}\right)$ be a bounded linear operator such that $T f$ is outer whenever $f$ is outer. Then, $T$ is necessarily a weighted composition operator, i.e. there exist analytic functions $a \in H^{q}\left(\mathbb{D}^{m}\right)$ and $b: \mathbb{D}^{m} \rightarrow \mathbb{D}^{n}$ such that $T f(z)=a(z) f(b(z))$ for every $z \in \mathbb{D}^{m}$ and $f \in H^{p}\left(\mathbb{D}^{n}\right)$.

Furthermore, $a=T 1$ is outer and $b=\frac{T(z)}{T 1}$, where $T(z)=\left(T\left(z_{i}\right)\right)_{i=1}^{n}$. 
Remark. (i) Note that the proof of Proposition 4.6 above is not valid for $0<q<1$ or $q=\infty$ since we use the duality of $L^{q}\left(\mathbb{T}^{m}\right)$ when $1 \leq q<\infty$. Therefore we do not obtain a result like Theorem 4.7 when $0<q<1$ or $q=\infty$. Theorem 4.13 is probably the best we can expect in these cases with our techniques.

(ii) It is not easy to determine when even a composition operator would preserve outer functions. One can check that in certain cases (like part (b) of Lemma 4.4.4 in [12]) the map $f \mapsto f \circ b$ preserves outer functions but more generally, it is not known to be true.

It would be interesting to characterize all weighted composition operators that preserve outer functions since it might help us understand the difference between outer functions and cyclic functions in $H^{p}\left(\mathbb{D}^{n}\right)$ for $n>1$. Unless, of course, all bounded weighted composition operators also preserve outer functions in which case we do not obtain any distinguishing property using cyclicity/outer preserving operators but rather, a kind of 'linear rigidity' between outer and cyclic functions. The following result shows that such a rigidity does not exist in general when $n>1$.

Theorem 4.14. Let $0<q<1 / 2$ be arbitrary. There exists a bounded linear map $T: H^{2}\left(\mathbb{D}^{2}\right) \rightarrow H^{q}(\mathbb{D})$ such that it preserves cyclicity, but not outer functions.

Proof. This example is from 12 but it was used in a different context; to obtain an outer function in $H^{2}\left(\mathbb{D}^{2}\right)$ which is not cyclic. We refer the reader to the discussion surrounding Theorem 4.4 .8 in 12 for proofs of the facts mentioned below.

Fix $0<q<1 / 2$. Let $T: H^{2}\left(\mathbb{D}^{2}\right) \rightarrow H^{q}(\mathbb{D})$ be defined as $T f(z)=f\left(\frac{1+z}{2}, \frac{1+z}{2}\right)$ for every $z \in \mathbb{D}$ and $f \in H^{2}\left(\mathbb{D}^{2}\right)$. Then $T$ is a bounded linear operator that preserves cyclicity. The function $f\left(z_{1}, z_{2}\right)=e^{\frac{z_{1}+z_{2}+2}{z_{1}+z_{2}-2}}$ is outer in $H^{2}\left(\mathbb{D}^{2}\right)$ but $T f(z)=e^{\frac{z+3}{z-1}}$ is not outer in $H^{q}(\mathbb{D})$ and so, $T$ does not preserve outer functions.

(iii) Notice that the proof of $(1) \Rightarrow(2)$ in Theorem 4.3 depends mostly on the properties of $\mathcal{X}$, since $\mathcal{Y}$ can be chosen to be fairly general. On the other hand, all the discussion about Hardy spaces shows that the proof of $(2) \Rightarrow(1)$ in Theorem 4.3 depends on the properties of $\mathcal{Y}$. In Proposition 4.6 , we saw that the proof relies heavily on the properties of $H^{p}\left(\mathbb{D}^{n}\right)$ and might not work for other spaces. This shows that while properties Q1-Q3 are quite reasonable for $\mathcal{X}$, it is not completely obvious what properties $\mathcal{Y}$ needs to have generally in order for the converse of Theorem 4.3 to hold true.

To show some different application of the abstract results proved in Sections 2 and 3, we conclude our discussion by proving a GKŻ-type theorem for spaces of analytic functions.

\section{GK $\dot{Z}$-type theorem for spaces of analytic functions}

The following result was proved independently by A. M. Gleason (Theorem 1, [6]), and J.-P. Kahane and W. Żelazko (Theorem 1, [7]).

Theorem 5.1. Let $\mathcal{B}$ be a complex unital Banach algebra, and let $\Lambda \in \mathcal{B}^{*}$ be such that $\Lambda(1)=1$. Then, $\Lambda(a b)=\Lambda(a) \Lambda(b)$ for every $a, b \in \mathcal{B}$ if and only if $\Lambda(a) \neq 0$ for every a invertible in $\mathcal{B}$.

We shall prove a similar result about partially multiplicative linear functionals on spaces of analytic functions as an interesting byproduct of topics discussed in Sections 2 and 3.

Definition 5.2. Suppose $\mathcal{X}$ is a space of functions that satisfies Q1-Q3 over $\hat{D} \subset \mathbb{C}^{n}$. We will consider two types of partially multiplicative linear functionals $\Lambda \in \mathcal{X}^{*}$ as follows. 
M1 For every $\phi \in \mathcal{M}(\mathcal{X}), f \in \mathcal{X}$ we have $\Lambda(\phi f)=\Lambda(\phi) \Lambda(f)$.

M2 For every $f, g \in \mathcal{X}$ such that $f g \in \mathcal{X}$ we have $\Lambda(f g)=\Lambda(f) \Lambda(g)$.

Note that if $\Lambda$ is $\mathbf{M 2}$, then $\Lambda$ is also M1. The converse need not be true in general. With this notation, the GKŻ-type theorem for spaces $\mathcal{X}$ that satisfies Q1-Q3 is as follows.

Theorem 5.3. Suppose $\widehat{\mathcal{X}}$ is a space of analytic functions on a set $\widehat{D} \subset \mathbb{C}^{n}$ that satisfies Q1-Q3. Let $\Lambda \in \mathcal{X}^{*}$ such that $\Lambda(1)=1$. Then, the following are equivalent :

(i) $\Lambda\left(e^{w \cdot z}\right) \neq 0$ for every $w \in \mathbb{C}^{n}$.

(ii) $\Lambda \equiv \Lambda_{b}$ for some $b \in \widehat{D}$.

(iii) $\Lambda$ is M2.

(iv) $\Lambda$ is $\mathbf{M 1}$.

Proof. Note that $(i) \Rightarrow(i i)$ follows from Theorem 3.5, and that $(I I) \Rightarrow(i i i) \Rightarrow(i v)$ is obvious from the definitions of M1 and M2 functionals.

For the proof of $(i v) \Rightarrow(i)$, assume $\Lambda$ is $\mathbf{M} 1$ and note that $e^{w \cdot z} \in \mathcal{M}(\widehat{\mathcal{X}})$ for every $w \in \mathbb{C}^{n}$. Thus,

$$
\Lambda\left(e^{w \cdot z}\right) \Lambda\left(e^{-w \cdot z}\right)=\Lambda\left(e^{w \cdot z} \cdot e^{-w \cdot z}\right)=\Lambda(1)=1
$$

for every $w \in \mathbb{C}^{n}$. Therefore $\Lambda\left(e^{w \cdot z}\right) \neq 0$ for every $w \in \mathbb{C}^{n}$.

We now show that the notion of maximal domains that we used in this paper comes from a class of partially multiplicative linear functionals.

Definition 5.4. Suppose $\mathcal{X}$ satisfies P1-P3. Let $\Lambda$ be a linear functional. We say $\Lambda$ is Mo if for every choice of polynomials $p$ and $q, \Lambda(p q)=\Lambda(p) \Lambda(q)$.

With this definition in mind, we have the following simple characterization of Mo functionals.

Proposition 5.5. Suppose $\mathcal{X}$ satisfies P1-P3 over an open set $D \subset \mathbb{C}^{n}$. Then, $\Lambda \in \mathcal{X}^{*}$ is $\mathbf{M O}$ if and only if $\left.\left.\Lambda\right|_{\mathcal{P}} \equiv \Lambda_{b}\right|_{\mathcal{P}}$ for some $b \in \sigma_{r}(S)$.

Proof. Suppose $\Lambda \in \mathcal{X}^{*}$ is M0. This means that for every $1 \leq i \leq n$ and $k \in \mathbb{N}, \Lambda\left(z_{i}^{k}\right)=\left(\Lambda\left(z_{i}\right)\right)^{k}$. Pick $b=\left(\Lambda\left(z_{i}\right)\right)_{i=1}^{n} \in \mathbb{C}^{n}$ so that $\Lambda(p)=p(b)$ for every polynomial $p$. To show $b$ lies in $\sigma_{r}(S)$ is the same as that in the proof of Theorem 2.1.

The converse is obvious since for every choice of polynomials $p$ and $q, \Lambda(p q)=p(b) q(b)=\Lambda(p) \Lambda(q)$.

What this means is that all reasonable notions of partially multiplicative linear functionals align when we consider these nice spaces of analytic functions, and Theorem 5.3 gives a way to describe these functionals in terms of their action on a certain set of exponential functions.

A similar result about different notions of partially multiplicative linear functionals on reproducing kernel Hilbert spaces with the complete Pick property was recently discovered (Corollary 3.4, 1]). It was proved that in the case of a complete Pick space, M1 and M2 are equivalent. It should be noted that this is not a 
special case of Theorem 5.3 since it covers Hilbert spaces of functions that are not necessarily analytic. On the other hand, Theorem 5.3 covers certain Banach spaces of analytic functions and not just Hilbert spaces.

It is also worth mentioning that just as we devised a maximal domain from Mo functionals on $\mathcal{X}$, one can construct a different notion of maximal domain from M1 and M2. Depending on what properties we want the extension $\hat{\mathcal{X}}$ to have, we may want to choose between M0, M1 and M2.

Since we were interested in preserving the behaviour of the shift operators and cyclic functions, Mo seemed appropriate as it respects multiplication of any function in $\mathcal{X}$ with any polynomial. If we were interested in a more algebraic behaviour as in 1], we would choose M2 instead since it is the strongest notion of partially multiplicative linear functionals we can expect from spaces of functions that are not algebras already. For a detailed discussion on this topic, refer to Section 2 in [10].

\section{Appendix}

In the proof of Theorem 2.1, we used it as a fact that all non-vanishing entire functions that satisfy a nice growth condition are of a specific type. This is not entirely obvious and we give a proof for it in this section. Since the proof of this fact is independent of all the other discussion, it seemed appropriate to present it separately.

Theorem 6.1. Fix $n \in \mathbb{N}$. Let $F \in \operatorname{Hol}\left(\mathbb{C}^{n}\right)$ be a non-vanishing entire function for which there exist constants $A, B$ such that $|F(z)| \leq A e^{B r^{m}}$ for all $z \in(r \mathbb{D})^{n}$ and for all $r>0$. Then, there exists a polynomial $p$ with $\operatorname{deg}(p) \leq m$ such that $F(z)=e^{p(z)}$ for all $z \in \mathbb{C}^{n}$.

Proof. Since $F$ is non-vanishing, there exists an entire function $G$ such that $F=e^{G}$. Note that the hypothesis then implies $\operatorname{Re}(G) \leq \ln A+B r^{m}$ in $(r \mathbb{D})^{n}$. Let $C:=\max \{\ln A, B\}+1$ so that $\operatorname{Re}(G)<C\left(1+r^{m}\right)$ in $\left(r \mathbb{D}^{n}\right)$. We need to show that this implies $G$ is a polynomial with $\operatorname{deg}(G) \leq m$.

First, consider the case $n=1$. Even though this is a well-known result, we provide a short proof for the sake of completion. Fix $r>0$ and define $g_{r}(z):=G(r z)$ for all $z \in \mathbb{C}$. By the hypothesis, $g_{r}$ maps the unit disc $\mathbb{D}$ into $\left\{\operatorname{Re}(w)<C\left(1+r^{m}\right)\right\}$ which is a left half-plane.

Using the Möbius transformation $\phi(z):=C\left(1+r^{m}\right)+\frac{z+1}{z-1}$ which maps $\mathbb{D}$ onto $\left\{\operatorname{Re}(w)<C\left(1+r^{m}\right)\right\}$, and applying Schwarz lemma to $\phi^{-1} \circ g_{r}$, we get

$$
\left|\frac{g_{r}(z)-g_{r}(w)}{2 C\left(1+r^{m}\right)-g_{r}(z)-\overline{g_{r}(w)}}\right| \leq\left|\frac{z-w}{1-z \bar{w}}\right|
$$

After plugging in $w=0$, clearing out the denominators, and using triangle inequality we get

$$
\left|g_{r}(z)-G(0)\right| \leq\left(2 C\left(1+r^{m}\right)+\left|g_{r}(z)\right|+|G(0)|\right) \cdot|z| \text { for every } z \in \mathbb{D}
$$

Fixing $|z|=1 / 2$, we get $\left|g_{r}(z)\right|-|G(0)| \leq\left|g_{r}(z)-G(0)\right| \leq C\left(1+r^{m}\right)+\frac{\left|g_{r}(z)\right|}{2}+\frac{|G(0)|}{2}$ which simplifies to

$$
|G(r z)| \leq 2 C\left(1+r^{m}\right)+3|G(0)| \text { whenever }|z|=1 / 2
$$

Since the choice of $r>0$ was arbitrary, we get that for every $z \in \mathbb{C}$,

$$
|G(z)| \leq 2 C\left(1+2^{m}|z|^{m}\right)+3|G(0)| .
$$


Choosing another appropriate constant $C_{0}$, we get $|G(z)| \leq C_{0}\left(1+r^{m}\right)$ for all $r>|z|$. If $G=\sum_{k \in \mathbb{N}} a_{k} z^{k}$ is the power-series of $G$, then using the above relation with Cauchy estimates for $a_{k}$ gives us $\left|a_{k}\right| \leq C_{1} / r^{k-m}$ for some constant $C_{1}$ and all $r>0$. Thus, $a_{k}=0$ for all $k>m$ which means $G$ is a polynomial with $\operatorname{deg}(G) \leq m$ as desired.

Assume now that $n>1$. Let $G(z)=\sum_{k \in \mathbb{N}} G_{k}(z)$ be the homogeneous expansion of $G$. Fix $z \in \mathbb{C}^{n}$ and let $g_{z}(\lambda):=G(\lambda z)=\sum_{k \in \mathbb{N}} \lambda^{k} G_{k}(z)$ for $\lambda \in \mathbb{C}$. Notice that

$$
\operatorname{Re}\left(g_{z}(\lambda)\right)=\operatorname{Re}(G(\lambda z)) \leq \ln A+B \cdot C^{m}|\lambda|^{m}
$$

where $C=\sup _{1 \leq j \leq n}\left|z_{j}\right|$, since $z \in(r \mathbb{D})^{n}$ for every $r>C$. Thus,

$$
\operatorname{Re}\left(g_{z}(\lambda)\right) \leq \ln A+B \cdot C^{m} r^{m}
$$

whenever $\lambda \in r \mathbb{D}$.

By the one variable case, we know that this implies $G_{k}(z)=0$ for all $k>m$. Since the choice of $z \in \mathbb{C}^{n}$ was arbitrary, this means $G_{k}(z)=0$ for all $z \in \mathbb{C}^{n}$ and $k>m$. Therefore $G$ is a polynomial with $\operatorname{deg}(G) \leq m$.

\section{References}

[1] Alexandru Aleman, Michael Hartz, John E McCarthy, and Stefan Richter, The Smirnov class for spaces with the complete Pick property, Journal of the London Mathematical Society 96 (2017), no. 1, 228-242.

[2] Catherine Bénéteau, Greg Knese, Łukasz Kosiński, Constanze Liaw, Daniel Seco, and Alan Sola, Cyclic polynomials in two variables, Transactions of the American Mathematical Society 368 (2016), no. 12, 8737-8754.

[3] Peter L Duren, Theory of $H^{p}$ spaces, Academic press, 1970.

[4] T. W. Gamelin, $H^{p}$ Spaces and Extremal Functions in $H^{1}$, Transactions of the American Mathematical Society 124 (1966), no. 1, 158-167.

[5] Peter C Gibson and Michael P Lamoureux, Constructive solutions to Pólya-Schur problems, Journal of Functional Analysis 269 (2015), no. 10, 3264-3281.

[6] Andrew M Gleason, A characterization of maximal ideals, Journal d'Analyse Mathématique 19 (1967), no. 1, $171-172$.

[7] J Kahane and W Żelazko, A characterization of maximal ideals in commutative Banach algebras, Studia Mathematica 29 (1968), 339-343.

[8] Kit Ian Kou and Junming Liu, Which linear operators preserve outer functions?, Indagationes Mathematicae 28 (2017), no. $6,1144-1156$.

[9] Javad Mashreghi and Thomas Ransford, A Gleason-Kahane-Żelazko theorem for modules and applications to holomorphic function spaces, Bulletin of the London Mathematical Society 47 (2015), no. 6, 1014-1020.

[10] John E McCarthy and Orr Moshe Shalit, Spaces of Dirichlet series with the complete Pick property, Israel Journal of Mathematics 220 (2017), no. 2, 509-530.

[11] Nikolai Nikolski, In a shadow of the RH: Cyclic vectors of Hardy spaces on the Hilbert multidisc, Annales de l'Institut Fourier 62 (2012), no. 5, 1601-1626 (en). MR3025149

[12] Walter Rudin, Function theory in polydiscs, Vol. 41, WA Benjamin, 1969. 